\title{
The influence of phylogenetic relatedness on species interactions among freshwater green algae in a mesocosm experiment
}

\author{
Patrick A. Venail ${ }^{1,2 *} \dagger$, Anita Narwani ${ }^{1}$, Keith Fritschie ${ }^{1} \ddagger$, Markos A. Alexandrou ${ }^{3}$, \\ Todd H. Oakley ${ }^{3}$ and Bradley J. Cardinale ${ }^{1}$ \\ ${ }^{1}$ School of Natural Resources and Environment, University of Michigan, Ann Arbor, MI 48109-1041, USA; ${ }^{2}$ Section of \\ Earth and Environmental Sciences, Institut F.-A. Forel, University of Geneva, 10 Route de Suisse, CP 416, 1290 \\ Versoix, Switzerland; and ${ }^{3}$ Department of Ecology, Evolution, and Marine Biology, University of California, Santa \\ Barbara, CA 93106-9620, USA
}

\section{Summary}

1. A long-standing hypothesis in ecology and evolutionary biology is that closely related species are more ecologically similar to each other and therefore compete more strongly than distant relatives do. A recent hypothesis posits that evolutionary relatedness may also explain the prevalence of mutualisms, with facilitative interactions being more common among distantly related species. Despite the importance of these hypotheses for understanding the structure and function of ecological communities, experimental tests to determine how evolutionary relatedness influences competition and facilitation are still somewhat rare.

2. Here, we report results of a laboratory experiment in which we assessed how competitive and facilitative interactions among eight species of freshwater green algae are influenced by their relatedness. We measured the prevalence of competition and facilitation among 28 pairs of freshwater green algal species that were chosen to span a large gradient of phylogenetic distances. For each species, we first measured its invasion success when introduced into a steady-state population of another resident species. Then, we compared its growth rate when grown alone in monoculture to its growth rate when introduced as an invader. The change in the species' population growth rate as an invader (sensitivity) is used as a measure of the strength of its interaction with the resident species. A reduced growth rate in the presence of another species indicates competition, whereas an increased growth rate indicates facilitation.

3. Although competition between species was more frequent ( $75 \%$ of interactions), facilitation was common (the other $25 \%$ of interactions). We found no significant relationship between the phylogenetic distance separating two interacting species and the success of invasion, nor the prevalence or strength of either competition or facilitation. Interspecific interactions depended more on the identity of the species, with certain taxa consistently acting as good or bad competitors/facilitators. These species were not predictable a priori from their positions on a phylogeny.

4. Synthesis. The phylogenetic relatedness of the green algae species used here did not predict the prevalence of competitive and facilitative interactions, rejecting the hypothesis that close relatives compete strongly and contesting recent evidence that facilitation is likely to occur between distant relatives.

Key-words: community phylogenetics, determinants of plant community diversity and structure, evolutionary ecology, green algae, invasion success, phylogenetic distance, sensitivity, species interactions

*Correspondence author. E-mail: Patrick.Venail@unige.ch $†$ Present address: Institut F.-A. Forel, University of Geneva, 10 Route de Suisse, CP 416, 1290 Versoix, Switzerland.

\$resent address: School of Aquatic and Fishery Sciences, University of Washington, Seattle, WA 98105, USA.

\section{Introduction}

Darwin proposed over 150 years ago that closely related species are more ecologically similar to each other than distantly 
related species (Darwin 1859). In turn, he argued that closely related species should compete more strongly and be less likely to coexist. For much of the last century, Darwin's hypothesis has been taken at face value by those who have proposed that conservation and restoration of evolutionary diversity could help maximize the stability (Cadotte, Dinnage \& Tilman 2012), resilience (Reusch et al. 2005), adaptability (Hoffmann \& Sgro 2011) and functioning of ecosystems (Cadotte, Cardinale \& Oakley 2008; Flynn et al. 2011; Venail \& Vives 2013). Despite its intuitive appeal and widespread use, the competition-relatedness hypothesis (hereafter abbreviated, $\mathrm{CRH}$ ) was not subjected to rigorous testing until recently, when the advent of molecular tools allowed us to better quantify the evolutionary relatedness of species.

We are aware of ca. 20 tests of Darwin's CRH that have been published over the past decade (Table 1). Of these, six (30\%) have found varying levels of support for the hypothesis that the strength of competition decreases as species become less related. Empirical tests to date have been performed using a variety of taxa, methodologies and metrics for measuring 'competition' (see Table 1), which has likely contributed to the varied results. It is also noteworthy that $55 \%$ of empirical tests to date (11 of 20 in Table 1) have found facilitative interactions among the species studied, accounting for up to $46 \%$ of all pairwise interactions (e.g. Fritschie et al. 2014). This is potentially problematic since the CRH was formulated to explain the coexistence of competing species and does not account for the evolution of mutualisms. Research on facilitation (i.e. neighbour or nurse effects in plants) suggests that, counter to expectations for competition, positive interactions may be more prevalent among distantly related species (Valiente-Banuet \& Verdú 2007, 2008; Castillo, Verdú \& Valiente-Banuet 2010). Thus, the prevalence of facilitative interactions in prior empirical tests of the $\mathrm{CRH}$ necessitates a more integrative hypothesis that accounts for how evolution influences both positive as well as negative interactions (Fig. 1). We propose that, as predicted by the $\mathrm{CRH}$, negative interactions among species are stronger among close relatives, and the strength and frequency of competitive interactions declines as the evolutionary distance among relatives increases. Given enough time, evolution may cause species to become sufficiently differentiated that they begin to use unique sets of resources, or have novel biological traits that can facilitate the nutritional needs of other species. The probability of facilitative interactions is more likely to occur with greater evolutionary divergence time, leading to the prediction that as phylogenetic distance increases, the strength of competition declines while the probability of facilitation increases (Fig. 1).

Here, we report the results of a study that tested the influence of phylogenetic relatedness on the prevalence of both competition and facilitation among 28 pairs of freshwater green algal species that span a large gradient in phylogenetic distances. To quantify species interactions, we ran pairwise invasibility experiments in which we introduced each species at low density (hereafter called 'the invader') into steady-state populations of the other species (hereafter called 'the resi- dent'). We tested whether the success of invasion was determined by the relatedness of the invader to the resident species. By comparing the growth rate of the invader when rare to the growth rate of that same species grown alone in monoculture, we quantified the sensitivity of each species to interactions with each of the other taxa (i.e. the proportional reduction or increase in growth rate; Carroll, Cardinale \& Nisbet 2011; Narwani et al. 2013). As we will show, species interactions among these algae varied from strong competition to facilitation; yet, neither the invasion success nor the interaction strengths were predicted by the evolutionary relationships among species.

\section{Materials and methods}

\section{SPECIES POOL}

We used eight species of freshwater green algae for this experiment (i.e. Chlorella sorokiniana, Closteriopsis acicularis, Cosmarium turpinii, Pandorina charkowiensis, Scenedesmus acuminatus, Selenastrum capricornotum, Staurastrum punctulatum and Tetraedron minimum). Cultures were obtained from collections at the University of Texas at Austin or the University of Gottingen (Germany). These eight taxa were chosen because, according to Environmental Protection Agency's 2007 Natural Lakes Assessment, they are widespread (i.e. present in at least $17 \%$ of over 1200 North American lakes sampled) and abundant (i.e. ranked among the top $50 \%$ of species densities of over 400 taxa). In addition, all eight species grow well under laboratory conditions using modified common growth media (COMBO enriched with $0.1 \mathrm{mM} \mathrm{KCl}$ and $30 \mu \mathrm{M} \mathrm{NH} \mathrm{NH}_{4} \mathrm{Cl}$ final concentrations) and can be morphologically distinguished under the microscope. Finally, this subset of species presented a relatively even distribution of phylogenetic distances among species sampled from a phylogenetic tree of North American freshwater algae (described in next section).

\section{PHYLOGENY}

To estimate the evolutionary relatedness among the eight species of algae, a molecular phylogeny including 37 North American freshwater green algal genera was generated using $18 \mathrm{~S}$ ribosomal rDNA and rbcl sequences extracted from GenBank and by including Guillardia theta, Porphyra torta and Cyanidioschyzon merolae as out-groups (see Figs S1 and S2 in Supporting Information). Sequences from Cosmarium turpinii were not available from GenBank, so we included two other representative Cosmarium species. Sequences for $P$. charkowiensis were also not available in GenBank, and so this species was represented by its closest available relative (Buchheim et al. 2011), Chlamydomonas reinhardtii. We constructed alignments independently for each gene using Muscle v 3.8.31 (Edgar 2004). Nucleotide substitution models were selected for each gene using the Akaike Information Criterion as implemented in jModeltest $\mathrm{v}$ 0.1.1 (Posada 2008), resulting in GTR + Gamma as the most likely model for both genes. We used this model to search for the maximumlikelihood phylogeny using RAxML v 7.2.8 (Stamatakis, Hoover \& Rougemont 2008), partitioning the data by gene. We used random starting trees for each independent tree search, and topological robustness was investigated using 100 nonparametric bootstrap replicates. We refer to resulting branch lengths as unsmoothed when they represent the average number of estimated substitutions per site in both genes. In addition, we calculated a rate smoothed Bayesian 


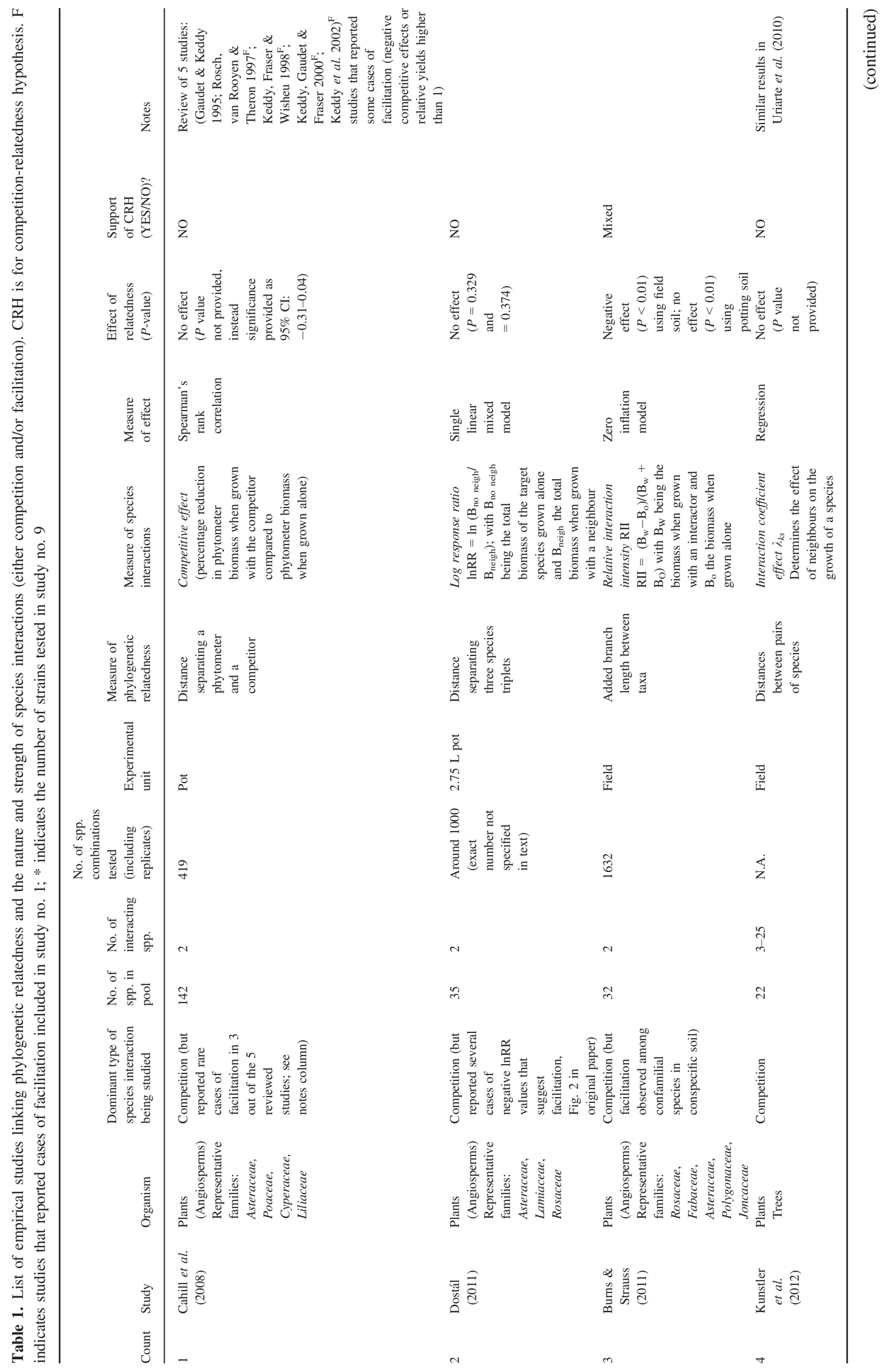




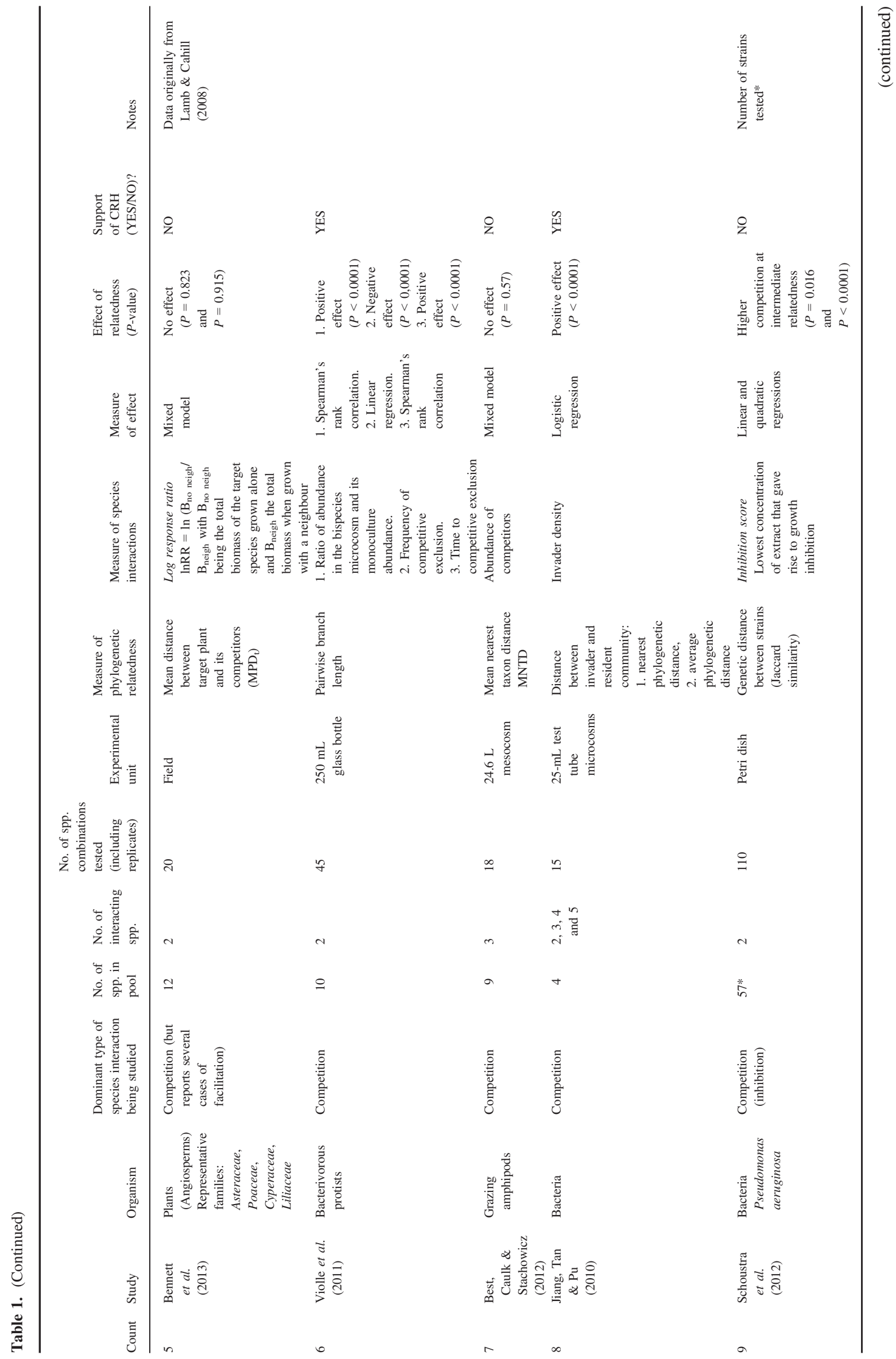




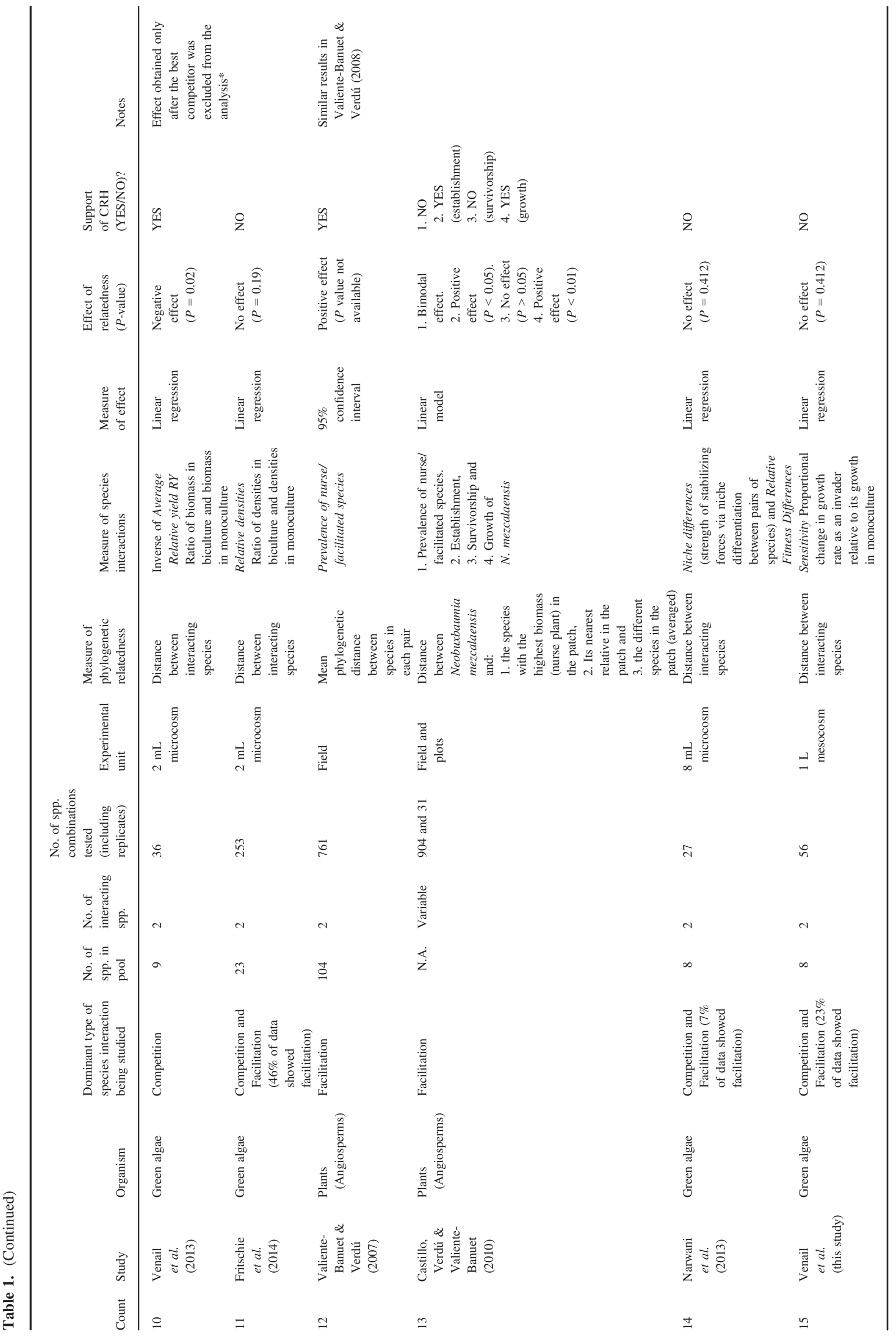



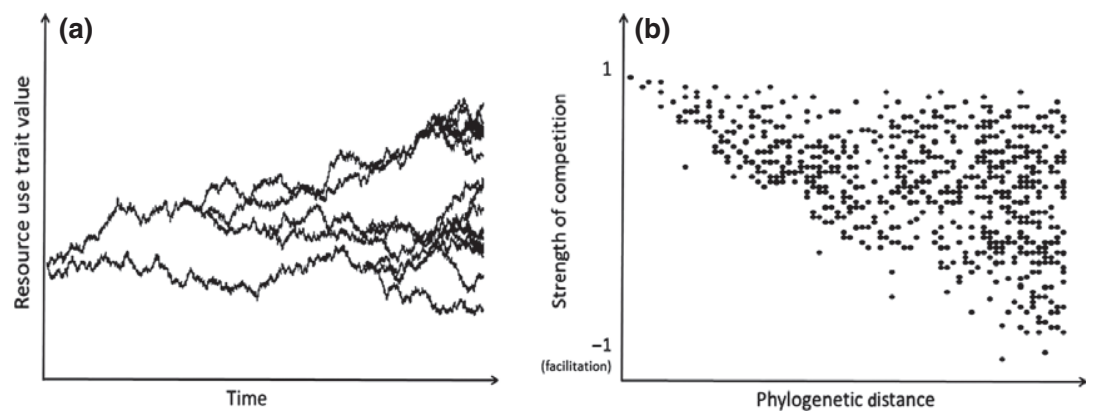

Fig. 1. We propose a modified version of Darwin's competition-relatedness hypothesis (CRH) that includes both positive as well as negative interactions; in (a) traits related to resource use and to competition diverge over time according to a Brownian motion random walk, which is a common null model of trait evolution. Species have some probability that traits diverge/converge at each time step, which causes variance in trait values, and trait differences to increase through time, on average. (b) If we randomly choose any two species from panel (a), the similarity in trait values separating these species and thus the strength of competition declines on average as phylogenetic distance increases (each dot is a pair of species from panel (a). Given enough time, evolution may cause species to become sufficiently differentiated that they begin to use unique sets of resources or have novel biological traits that can facilitate the nutritional needs of other species. The probability of facilitative interactions is more likely to occur with greater evolutionary divergence time, leading to the prediction that as phylogenetic distance increases, the strength of competition declines while the probability of facilitation increases.

phylogeny, estimated using BEAST v1.6.2 (Drummond \& Rambaut 2007), assuming a relaxed uncorrelated log-normal clock with all other parameters on default. We conducted the relaxed clock analysis to estimate relative divergence times, thereby converting branch length values from substitutions per site to an estimate of time since divergence from a common ancestor. The Bayesian MCMC chain ran for 10 million generations samples every 1000 generations, while stationarity and effective sample sizes (ESS $>200$ ) were estimated using Tracer v1.5 (Rambaut \& Drummond 2009), discarding all trees under the asymptote as burn-in. We constructed a consensus tree using TreeAnnotator v1.6.2 (Drummond \& Rambaut 2007). We then calculated the phylogenetic distance between each pair of species as the sum of tree branch lengths connecting them (Cadotte, Cardinale \& Oakley 2008) using a custom Bioperl (Stajich et al. 2002) script from mean branch lengths (of all bootstrap pseudoreplicates for maximum likelihood and for all tress retained from the MCMC search for Bayesian analyses) connecting each species pair and ignoring the root branch. For Cosmarium turpinii, we calculated the mean of the phylogenetic distances of its two congeners.

\section{EXPERIMENTAL DESIGN}

We prepared 192 1-L sterile Pyrex glass bottles filled with 1L of enriched COMBO growth medium. Each of the eight algal species was inoculated as monocultures into 24 bottles. Bottles were inoculated at an initial density of 200 cells $\mathrm{mL}^{-1}$ and placed on Wheaton ${ }^{\circledR}$ (349000-A) roller racks and cultured at $20^{\circ} \mathrm{C}$ under a $16: 8$ hour light/dark cycle at a light intensity of ca. $81 \mu \mathrm{E} \mathrm{m}^{-2} \mathrm{~s}^{-1}$. Three bottles of each species were maintained as monocultures for the duration of the experiment (i.e. were never invaded). The remaining 21 bottles were invaded by one of each of the seven other species in the species pool, with each invader treatment being replicated three times. Fresh growth medium was replaced every other day with a $10 \%$ exchange rate $(100 \mathrm{~mL})$ starting 4 days after the initial inoculation. We measured fluorescence of chlorophyll-a as proxy for algal biomass on a well-plate reader (Fluorometer, Synergy H1 Hybrid Reader, Biotek) every second day for the first month of the study. This allowed us to track the growth dynamics of the cultures. We waited for all of the cultures to achieve steady state before adding the invading species on day 28 of the experiment. Invaders were also inoculated at a density of 200 cells per $\mathrm{mL}$, and they were introduced with replacement sterile medium on a media-exchange day. Media exchanges and sampling started again two days after invasion and continued for 12 additional days.

\section{DATA COLLECTION AND ANALYSIS}

Twelve days after cultures were inoculated for the first time, the three control bottles of each species (i.e. monocultures without invasion) were sampled $(1 \mathrm{~mL})$ to estimate growth rates of monocultures in the absence of competitors. Similarly, 12 days after invasion (i.e. day 40 of the experiment), $1 \mathrm{~mL}$ aliquots were collected from every bottle to estimate growth rates of invaders. All samples were fixed by adding $250 \mu \mathrm{L}$ of $10 \%$ formalin to stop growth and preserve cells and stored in the dark for later analysis. We measured growth rates over twelve days because preliminary data on monoculture growth curves indicated this time period maximized the likelihood of detecting growth for slow-growing species while minimizing the potential for densitydependent feedbacks for fast-growing species. All samples were counted on a haemacytometer using a compound light microscope. The density of monocultures (i.e. control bottles) and invader species' densities were estimated by counting 400 cells or 10 full haemacytometer grids $(9 \mu \mathrm{L})$, whichever occurred first. Cell counts were converted to cell densities by accounting for the volume of sample searched. Growth rates were calculated as:

$g=(1 / T) * \ln \left(D_{T} / D_{0}\right)$

eqn 1

where $T$ is 12 days, $D_{T}$ is the density of the monoculture (i.e. in control bottle) or invader on day 12 and $D_{0}$ is the inoculated cell density on day 0 (i.e. 200 cells per $\mathrm{mL}$ ).

\section{Invasion success}

Positive growth rates $(g>0)$ as invaders indicated successful invasion of the resident's steady-state population (recorded as 1 ), whereas negative growth rates $(g<0)$ indicated unsuccessful invasion (recorded as 0). In total, 168 different values of either successful or unsuccessful invasion were included in our first analysis (i.e. 28 bicultures, two directions: either species A invading species B or 
species B invading species A, replicated three times each). We used logistic regression to assess the influence of phylogenetic distance on invasion success.

\section{Sensitivity}

A species' $i$ sensitivity $\left(S_{i}\right)$ of growth rate to the presence of a resident alga was measured as the proportional change in its growth rate as an 'invader' relative to its growth in monoculture (Carroll, Cardinale \& Nisbet 2011):

$S_{i}=\left(g_{\text {i mono }}-g_{i \text { invader }}\right) /\left(g_{\text {i mono }}\right)$ eqn 2

Where $g_{i \text { mono }}$ is the growth of species $i$ in monoculture and $g_{i \text { invader }}$ is the growth of species $i$ as an invader of an established population. Growth rates were averaged over the three replicates for each treatment. None of the results presented here changed when using replicates as individual points. When $S_{i}$ is higher than one (negative growth of $i$ as invader), it means that invasion was unsuccessful and the invader species $i$ failed to establish, suggesting strong competition and high niche overlap between the invader and the established species. As the value of $S_{i}$ approaches 1 (but lower than 1), the species $i$ grows less well as an invader than it does in monoculture, suggesting a negative impact of competition. When $S_{i}$ approaches zero (but remain positive), species $i$ grows as well as an invader as when growing by itself, suggesting that the species is not negatively affected by competition and niche overlap with the established species is low. Finally, a negative sensitivity means that the species grows better as an invader than by itself in monoculture and can be interpreted as evidence of facilitation. An originality of our approach is that compared to previous studies (Table 1), our system allowed us estimating competitive and facilitative interactions (i.e. sensitivity) by using changes on population growth rates over long periods of time. We calculated 135 sensitivity values among the species combinations after 33 of the initial 168 samples were eliminated from consideration because the invader was not detected in the samples on the final day of the experiment. Without estimates of growth rates, it was not possible to calculate $S_{i}$ values for these 33 combinations. We used linear regression to test whether values of sensitivity depended on phylogenetic distance after testing for homoscedasticity of data using a studentized Breush Pagan test $(B P=2.331$, d.f. $=1, P=0.1268)$. We used logistic regression to test for an effect of phylogenetic distance on the prevalence of facilitative (i.e. negative sensitivities) or competitive interactions (i.e. positive sensitivities).

We determined whether any species was involved in facilitative interactions or competition more often than would be expected by chance by performing a chi-square test on the prevalence of facilitation (i.e. negative sensitivities) and competition (i.e. positive sensitivities). First, we grouped data per invading species, for instance species A (i.e. Chlorella sorokiniana) invading the other seven species (named $\mathrm{X}$ for simplicity). We did the same for B invading $\mathrm{X}, \mathrm{C}$ invading $\mathrm{X}$, etc. This test would reveal whether any species was being facilitated by or was competing with the established species more often than expected by chance. Secondly, we grouped the data per species being invaded, for instance species A (i.e. Chlorella sorokiniana) being invaded by the other seven species (named $\mathrm{Y}$ for simplicity). We did the same for B being invaded by $\mathrm{Y}, \mathrm{C}$ being invaded by $\mathrm{Y}$, etc. This test would reveal, for instance, whether any species was acting as facilitator or was competing with the invader more often than expected by chance. Additionally, we determined how a given species' growth rate as invader was impacted by the presence of a resident algal species and also the impact that it had as resident on the other species' growth as invaders. As a result, for each species, we analysed sensitivity values in two ways, analogous to Goldberg's 'competitive effect and response' (Goldberg \& Fleetwood 1987): A) Sensitivity as the invader species: For any given species $i$, we averaged the sensitivity of $i$ when invading species $j-p$ to reveal whether a species could, or could not, invade other species. Good invaders would have average sensitivities as invaders close to 0 , whereas bad invaders would have average sensitivity as invaders close to 1 . Species with negative sensitivities as invaders may have benefited from facilitation by other species. B) Effect on invader sensitivities as the resident: For any given species $i$, we averaged the sensitivity of species $j-p$ invading $i$ to assess whether or not $i$ was easily invaded by other species. An average sensitivity as resident close to 0 suggests that the species can be easily invaded, whereas an average sensitivity as resident close to 1 suggests that the species is difficult to invade. An average negative sensitivity as resident suggests the species is a facilitator. Using the average sensitivities as invaders and the average effects on sensitivities as residents, we ranked the eight species as good or bad competitors and as facilitators or facilitated species. Good competitors' growth was not affected by the presence of the resident species (sensitivity as invader close to 0) but strongly decreased invader's growth (effect on invader's sensitivity close to 1 ). Bad competitors' growth was heavily affected by the presence of the resident species (sensitivity as invader close to 1) and did not decrease invader's growth (effect on invader's sensitivity close to 0 ). Facilitators would help other species to perform better than in monoculture, whereas facilitated species would grew better in the presence of other species than alone.

\section{Results}

\section{INVASION SUCCESS}

Of the 168 invasibility tests performed in this study, invasion was successful 114 times (Table S2), as determined from positive growth rates over the 12 days following inoculation. Invasion was unsuccessful 54 times with negative growth rates over the 12 days following inoculation, including the 33 cases where the invader was not detected in the culture 12 days after inoculation. There was a slightly negative relationship between phylogenetic distance among species and the probability of successful invasion (Fig. 2, logistic regression, $\chi^{2}=0.02, P=0.06, n=168$ ). Regardless of whether or not the relationship is significant, it is exactly the opposite of what would be expected if (a) more distantly related species experience less competition and (b) more distantly related species have a higher probability of facilitative interactions (Fig. 1).

\section{NATURE AND STRENGTH OF INTERSPECIFIC INTERACTIONS}

Evidence of competition among species was over three times more frequent than evidence of facilitation. Out of the 135 estimates of sensitivity, 31 were negative, indicating the presence of facilitation, and 104 were positive, indicating the presence of competition (or 137 if we include the 33 cases where the invader was not detected twelve days after inoculation). Neither the prevalence of facilitation (negative 


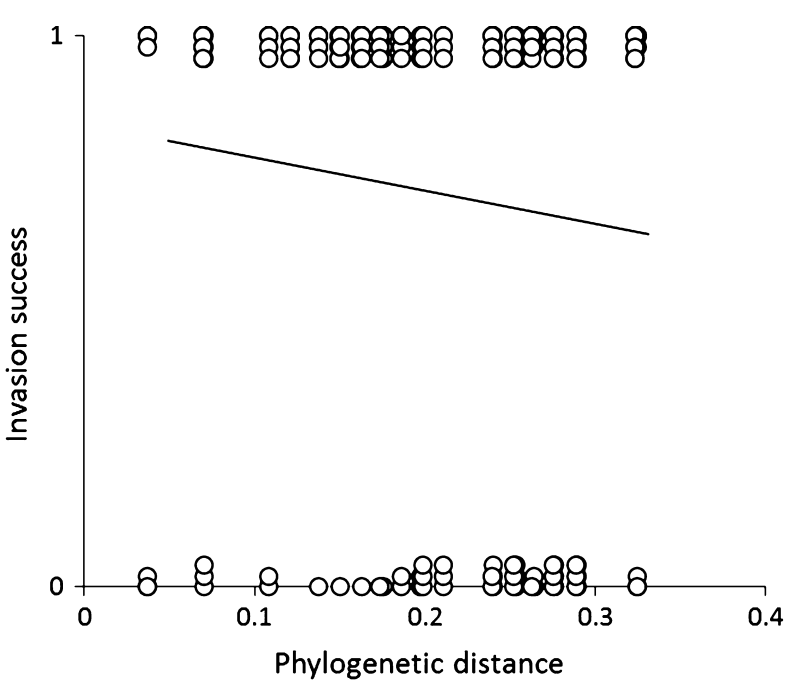

Fig. 2. Effect of phylogenetic distance (i.e. relatedness between two species) on the successfulness of invasion (successful invasion $=1$, unsuccessful invasion $=0$ ). Each dot represents a pairwise species combination. The line represents the logistic regression between phylogenetic distance and prevalence of invasibility which in this case is not significant (or slightly significant, depending on $P$-value criteria, $P=0.06$ ). For visual clarity, superposed dots (same phylogenetic distance among species) were slightly displaced along the $y$-axis.

sensitivities) nor competition (positive sensitivities) was a function of the phylogenetic distance between any two species (Fig. 3a, $\chi^{2}=0.005, P=0.412, n=135$ ). Results were similar when including the 33 cases where invasion was not successful $\left(\chi^{2}=0.004, P=0.362, n=168\right)$. Indeed, facilitative interactions were observed across the full gradient of phylogenetic distances investigated in this study and were prevalent among both close and distant relatives. The same was true for competitive interactions. Similarly, we found that the evolutionary relatedness of species (i.e. phylogenetic distance) had no influence on the sensitivity of growth rates to species interactions (Fig. 3b, linear regression, $r^{2}=0.0004, \quad P=0.82$, $n=135)$.

Although there was no phylogenetic signal in competition (Fig. 3), there was considerable variability among species in their sensitivity to interactions, and there was consistency in which species were good or bad competitors (Fig. 4) and which species were involved in competitive interactions (Table S1). Chlorella sorokiniana was on average the best competitor, being the most difficult species to invade (top of $y$-axis in Fig. 4) with an average sensitivity as resident close to unity, suggesting invaders were not able to grow when Chlorella sorokiniana was already established in the culture. Chlorella sorokiniana and Scenedesmus acuminatus were on average the best invaders (left side of $x$-axis in Fig. 4), with their average sensitivities as invaders close to zero, suggesting they grew just as well as invaders as they did in monoculture. Three other species Closteriopsis acicularis, Scenedesmus acuminatus and Selenastrum capricornotum were also relatively difficult to invade with average effects on sensitivities as residents above 0.5 . Even so, these taxa ranged from very good (Scenedesmus acuminatus) to good (Closteriopsis acicularis)
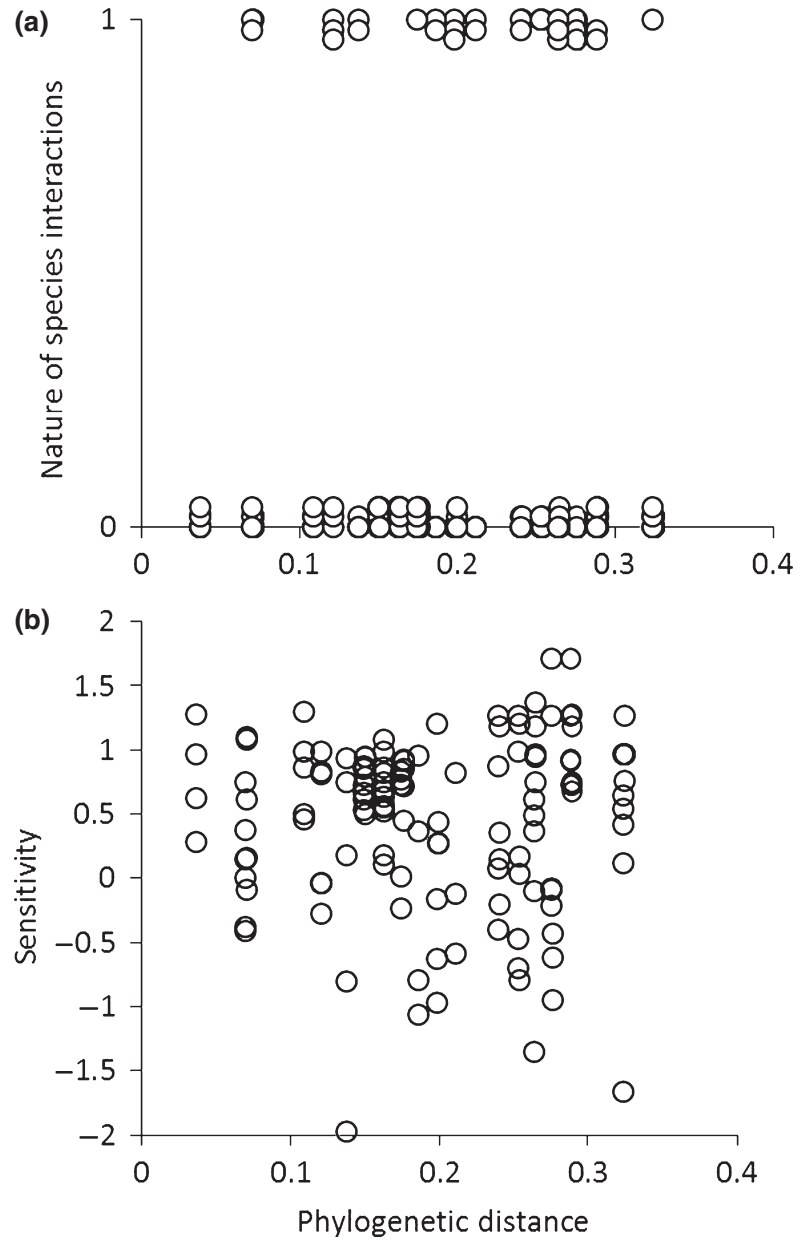

Fig. 3. Effect of phylogenetic distance on the nature of a) species interactions and b) sensitivity. In (a), either facilitation ( 1 on the $y$-axis) or competition ( 0 on the $y$-axis) is represented. The line represents the logistic regression between phylogenetic distance and prevalence of facilitation which in this case is not significant. For visual clarity, superposed dots (same phylogenetic distance among species) were slightly displaced along the $y$-axis. (b) Effect of phylogenetic distance on sensitivity. In both panels, each dot represents a pairwise species combination.

and poor invaders (Selenastrum capricornotum). Pandorina charkowiensis was on average the worst competitor, with sensitivity as invader close to 1 , suggesting its inability to invade an already established population. It was also the easiest species to invade (bottom of $y$-axis in Fig. 4) with invaders being able to grow in its presence as well as they grew in monoculture (average effect on sensitivity as resident close to zero). Cosmarium turpinii, Staurastrum punctulatum and T. minimum were relatively easy to invade with average effects on sensitivities as residents below 0.5. Cosmarium turpinii, P. charkowiensis, Selenastrum capricornotum, Staurastrum punctulatum and T. minimum competed with the resident species more often than expected by chance (chi-square test, d.f. $=7$, $\chi^{2}=52.176, P<0.0001, n=168$, Table S1a). Chlorella sorokiniana, Closteriopsis acicularis, Scenedesmus acuminatus and Selenastrum capricornotum competed with the invading species more often than expected by chance (chi-square test, d.f. $=7$, $\chi^{2}=25.594, P=0.0006, n=168$; see Table $\left.\mathrm{S} 1 \mathrm{~b}\right)$. 


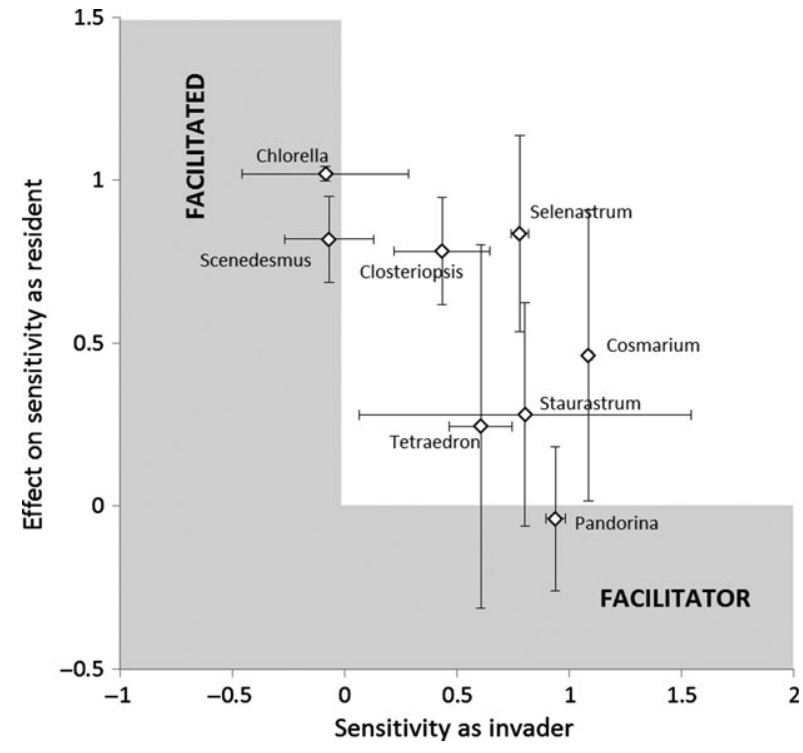

Fig. 4. Average sensitivity as invader and effect on sensitivity as focal species for each of the eight species included in our study. The $x$-axis represents how good a species was at invading a steady-state population of other algal taxa with values close to 0 for good invaders and values close to 1 for bad invaders. The $y$-axis represents how resistant to invasion a focal species was with values close to 0 for species easy to invade and values close to 1 for species difficult to invade. Overall, good competitors are located at the left-upper corner (e.g. Chlorella), while bad competitors are located at the right-lower corner (e.g. Pandorina). Two facilitation zones are depicted: facilitators have effects on sensitivities as residents lower than 0 (horizontal shaded zone), whereas facilitated species have sensitivities as invaders lower than 0 (vertical shaded zone). The unshaded zone represents competition. Bars represent standard errors. Only genera names are presented (see main text for complete species names).

Despite the lack of phylogenetic signal in the prevalence of facilitation (Fig. 3a), some species showed evidence of being involved in facilitative interactions more often than expected by chance, either by being facilitated by other species (chisquare test, d.f. $=7, \chi^{2}=52.176, P<0.0001, n=168$, Table S1a) or by facilitating other species (chi-square test, d.f. $=7, \quad \chi^{2}=25.594, \quad P=0.0006, n=168$, Table S1b). Chlorella sorokiniana, Closteriopsis acicularis and particularly Scenedesmus acuminatus were very often facilitated by other species, whereas Cosmarium turpinii, P. charkowiensis and Selenastrum capricornotum were never facilitated. Four species acted as facilitators more often than expected by chance: Cosmarium turpinii, P. charkowiensis, Staurastrum punctulatum and T. minimum. On the contrary, Chlorella sorokiniana and Scenedesmus acuminatus never acted as facilitators.

\section{Discussion}

A common assumption made by several studies incorporating evolutionary information into community ecology research is that the nature and strength of species interactions depends on the phylogenetic relatedness of species, with competition being strong among close relatives and facilitation occurring primarily among distantly related taxa (Fig. 1). Results of our mesocosm experiments using a pool of eight species of freshwater green algae showed that neither competitive nor facilitative interactions depended on the evolutionary relatedness of interacting species for this group of organisms.

Our results add to a growing body of literature that fails to support Darwin's original competition-relatedness hypothesis (Table 1). This suggests that one or more of the underlying assumptions of this hypothesis (Fig. 1) do not hold true for freshwater green algae. One assumption of the competitionrelatedness hypothesis is that the phylogenetic relatedness of species is positively related to their ecological similarity with traits influencing competitive interactions being phylogenetically conserved (Mayfield \& Levine 2010). A second assumption is that ecologically similar species compete more strongly than less ecologically similar species (e.g. limiting similarity hypothesis, MacArthur \& Levins 1967). This assumption ignores the possibility of competition being driven by fitness differences among species and not just by niche differences alone (Adler, HilleRisLambers \& Levine 2007; Mayfield \& Levine 2010). Violation of either of these assumptions would result in phylogenetic history having no influence on the strength of competitive interactions, as observed here. In a recent study supporting the competitionrelatedness hypothesis, Violle and collaborators (2011) found that competitive exclusion occurred more frequently and more rapidly between closely related species of bacterivorous protists, for which a key competition-related trait (i.e. mouth size) was phylogenetically conserved. However, while examples of niche conservatism exist, this phenomenon has proven to be far from ubiquitous. Some studies have shown that competitive traits are phylogenetically conserved (Slingsby \& Verboom 2006; Swenson et al. 2007; Kraft \& Ackerly 2010; Violle et al. 2011; Maherali \& Klironomos 2012), whereas others have shown they are evolutionarily labile (Prinzing et al. 2001; Losos et al. 2003; Losos 2008; Liu et al. 2012). Thus, the lack of phylogenetic signal on competition-related traits often undermines the assumption that phylogenetic distance can be used as a proxy for species similarity (Thuiller et al. 2010; Mouquet et al. 2012).

Although we found evidence of facilitation in roughly onequarter of the pairwise species interactions, the prevalence of this form of interaction did not depend on the evolutionary relatedness of species. This result contradicts evidence from recent field studies suggesting that the prevalence of facilitative interactions tends to be most common between distantly related species (Valiente-Banuet \& Verdú 2007, 2008; Castillo, Verdú \& Valiente-Banuet 2010; see Verdu, GomezAparicio \& Valiente-Banuet 2012 for a review). By classifying over 450 angiosperm species as 'non-facilitated' (i.e. species recruiting on open ground) or 'facilitated' (i.e. species recruiting under vegetation), Valiente-Banuet \& Verdú (2007) determined that a trait they called the 'regeneration niche' was strongly conserved. As a consequence of phylogenetic conservatism of the regeneration niche, nurse plants more frequently facilitate distantly related species than closely related species (Valiente-Banuet \& Verdú 2007). Under the assump- 
tion that traits related to facilitation are evolutionarily conserved, it is expected that the prevalence of facilitative interactions will increase with phylogenetic distance. In accordance with this trend, Castillo, Verdú and Valiente-Banuet (2010) found that the performance of a cactus (Neobuxbaumia mezcalensis) was positively influenced by the presence of neighbour 'nurse' plants and this positive effect increased as the neighbours were less related to the cactus. Similarly, Verdu, Gomez-Aparicio and Valiente-Banuet (2012) found that the less related the neighbouring plants were to a focal plant, the greater the positive effect on the nurse plant growth. However, in an observational study carried out in Spanish steppes (Soliveres, Torices \& Maestre 2012), the effect of relatedness on the prevalence of facilitative interactions among species proved to be more mixed. The growth of the grass Stipa tenacissima was not affected by relatedness of its neighbours. The growth of a shrub Quercus coccifera was negatively influenced by the presence of nurse species within a range of relatedness of 207-273 million years and facilitated by the presence of closer or more distantly related species. As may be true for traits related to competition, it is possible that the traits involved in facilitative interactions are not phylogenetically conserved in the green algae included in our experiment.

Recently, some progress has been made depicting the functional traits controlling competition and community structure in freshwater phytoplanktonic systems (Litchman et al. 2010; Edwards, Klausmeier \& Litchman 2011; Schwaderer et al. 2011). For instance, when considering a very large range of algal taxonomic groups, there is evidence that competitive abilities for nitrate and phosphate are negatively correlated, suggesting that species performing well under nitrate limited conditions perform badly under phosphate limited conditions and vice versa (Edwards, Klausmeier \& Litchman 2011). Moreover, traits linked to light utilization and maximal growth rates have been successfully used to predict phytoplanktonic community structure across U.S. lakes (Edwards, Litchman \& Klausmeier 2013). For these large taxonomic groupings, cell size may also act as a master trait influencing phytoplankton community structure, with large-celled taxa having an advantage under the nutrient-abundant or nutrientfluctuating conditions and small taxa being favoured under nutrient-restricted conditions (Litchman et al. 2010; Edwards, Klausmeier \& Litchman 2011). Unfortunately, the phylogenetic signal of traits related to competition and facilitation has not yet been conducted.

Overall, the evolution of facilitative traits remains minimally explored (Bronstein 2009) and the functional traits responsible for facilitative interactions in plants are being investigated (Butterfield \& Callaway 2013). While there has been some work done already to identify the traits responsible for the outcome of competition in freshwater algae (Tilman 1981; Edwards, Litchman \& Klausmeier 2013), there has been virtually no work investigating facilitative or mutualistic interactions in these species. Without direct evidence, our hypotheses regarding these traits are currently speculative. Facilitative interactions may involve the ability of one species to provide resources such as vitamins or other organic molecules (produced as metabolites) to another in the form of cross-feeding relationships. Some species may also modify water chemistry (e.g. $\mathrm{pH}$ or dissolved $\mathrm{CO}_{2}$ concentrations) or light availability in a beneficial way for their competitors. Testing the mechanisms by which Scenedesmus acuminatus and Chlorella sorokiniana benefited from the presence of other algae in this study will be an avenue for future work.

The lack of universality of niche conservatism, the absence of phylogenetic signal in traits relevant for competition and/or facilitation, and the limited support for the competition-relatedness hypothesis illustrate some of the difficulties and limitations of integrating phylogenetic information into community ecology research. The incorporation of a phylogenetic perspective into community ecology and ecosystem functioning research was initially based at least partially on the relative ease of measuring phylogenetic distance compared to measures of functional differentiation. Phylogenetic distance was hypothesized to represent a cheap and reliable metric capable of summarizing all of the ecological differences among species. Based on the results observed here and along with previous studies, we have no reason to believe phylogenetic distance alone should generally predict the outcome of competition or facilitative interactions. Our results suggest that the phylogenetic relatedness of species may not be a reliable proxy for functional or ecological similarity and may not be used to infer the forces determining the structure of ecological systems. Although, additional studies relating the nature and strength of species interactions to the evolutionary relatedness of species of other taxa and the investigation of this relationship at other phylogenetic scales are required to validate the generality of our findings.

\section{Acknowledgements}

We thank S. Beaudry, K. Kulacki, M. McCarthy, E. Mitchell, P. Parent, C. Wilson and C. Zhou for technical assistance during the experiment. This work was supported by the U.S. National Science Foundation's DIMENSIONS of Biodiversity programme in a grant to B.J.C. (DEB-1046121) and to T. H. O. (DEB-1046121).

\section{References}

Adler, P.B., HilleRisLambers, J. \& Levine, J.M. (2007) A niche for neutrality. Ecology Letters, 10, 95-104.

Bennett, J.A., Lamb, E.G., Hall, J.C., Cardinal-McTeague, W.M. \& Cahill, J.F. $\mathrm{Jr}$ (2013) Increased competition does not lead to increased phylogenetic overdispersion in a native grassland. Ecology Letters, 16, 1168-1176.

Best, R.J., Caulk, N.C. \& Stachowicz, J.J. (2012) Trait vs. phylogenetic diversity as predictors of competition and community composition in herbivorous marine amphipods. Ecology Letters, 16, 72-80.

Bronstein, J.L. (2009) The evolution of facilitation and mutualism. Journal of Ecology, 97, 1160-1170.

Buchheim, M.A., Keller, A., Koetschan, C., Förster, F., Merget, B. \& Wolf, M. (2011) Internal transcribed spacer 2 (nu ITS2 rRNA) sequence-structure phylogenetics: towards an automated reconstruction of the green algae tree of life. PLOS ONE, 6, e16931.

Burns, J.H. \& Strauss, S.Y. (2011) More closely related species are more ecologically similar in an experimental test. Proceedings of the National Academy of Sciences, 108, 5302-5307.

Butterfield, B.J. \& Callaway, R.M. (2013) A functional comparative approach to facilitation and its context dependence. Functional Ecology, 27, 907-917. 
Cadotte, M.W., Cardinale, B.J. \& Oakley, T.H. (2008) Evolutionary history and the effect of biodiversity on plant productivity. Proceedings of the National Academy of Sciences, 105, 17012-17017.

Cadotte, M.W., Dinnage, R. \& Tilman, D. (2012) Phylogenetic diversity promotes ecosystem stability. Ecology, 93, S223-S233.

Cahill, J.F. Jr, Kembel, S.W., Lamb, E.G. \& Keddy, P.A. (2008) Does phylogenetic relatedness influence the strength of competition among vascular plants? Perspectives in Plant Ecology, Evolution, and Systematics, 10, 4150 .

Carroll, I.T., Cardinale, B.J. \& Nisbet, R.M. (2011) Niche and fitness differences relate the maintenance of diversity to ecosystem function. Ecology, $\mathbf{9 2}$, $1157-1165$

Castillo, J.P., Verdú, M. \& Valiente-Banuet, A. (2010) Neighborhood phylodiversity affects plant performance. Ecology, 91, 3656-3663.

Darwin, C. (1859) On the Origin of Species by Means of Natural Selection. John Murray, London.

Dostál, P. (2011) Plant competitive interactions and invasiveness: searching for the effects of phylogenetic relatedness and origin on competition intensity. The American Naturalist, 177, 655-667.

Drummond, A.J. \& Rambaut, A. (2007) BEAST: Bayesian evolutionary analysis by sampling trees. BMC Evolutionary Biology, 7, 214

Edgar, R.C. (2004) MUSCLE: a multiple sequence alignment method with reduced time and space complexity. BMC Bioinformatics, 5, 1-19.

Edwards, K.F., Klausmeier, C.A. \& Litchman, E. (2011) Evidence for a threeway trade-off between nitrogen and phosphorus competitive abilities and cel size in phytoplankton. Ecology, 92, 2085-2095.

Edwards, K.F., Litchman, E. \& Klausmeier, C.A. (2013) Functional traits explain phytoplankton responses to environmental gradients across lakes of the United States. Ecology, 94, 1626-1635.

Flynn, D.F.B., Mirotchnick, N., Jain, M., Palmer, M.I. \& Naeem, S. (2011) Functional and phylogenetic diversity as predictors of biodiversity-ecosystem-function relationships. Ecology, 92, 1573-1581.

Fritschie, K.J., Cardinale, B.J., Alexandrou, M.A. \& Oakley, T. (2014) Evolutionary history and the strength of species interactions: testing the phylogenetic limiting similarity hypothesis. Ecology, 95, 1407-1417.

Gaudet, C.L. \& Keddy, P.A. (1995) Competitive performance and species distribution in shoreline plant communities: a comparative approach. Ecology, 76, 280-291.

Goldberg, D.E. \& Fleetwood, L. (1987) Competitive effect and response in four annual plants. Journal of Ecology, 75, 1131-1143.

Hoffmann, A.A. \& Sgro, C.M. (2011) Climate change and evolutionary adaptation. Nature, 470, 479-485.

Jiang, L., Tan, J. \& Pu, Z. (2010) An experimental test of Darwin's naturalization hypothesis. The American Naturalist, 175, 415-423.

Keddy, P., Fraser, L.H. \& Wisheu, I.C. (1998) A comparative approach to examine competitive response of 48 wetland plant species. Journal of Vegetation Science, 9, 777-786.

Keddy, P., Gaudet, C. \& Fraser, L.H. (2000) Effects of low and high nutrients on the competitive hierarchy of 26 shoreline plants. Journal of Ecology, 88, 413-423.

Keddy, P., Nielsen, K., Weiher, E. \& Lawson, R. (2002) Relative competitive performance of 63 species of terrestrial herbaceous plants. Journal of Vegetation Science, 13, 5-16.

Kraft, N. \& Ackerly, D.D. (2010) Functional trait and phylogenetic tests of community assembly across spatial scales in an Amazonian forest. Ecological Monographs, 80, 401-422.

Kunstler, G., Lavergne, S., Courbaud, B., Thuiller, W., Vieilledent, G., Zimmermann, N.E., Kattge, J. \& Coome, D.A. (2012) Competitive interactions between forest trees are driven by species' trait hierarchy, not phylogenetic or functional similarity: implications for forest community assembly. Ecology Letters, 15, 831-840.

Lamb, E.G. \& Cahill, J.F. Jr (2008) When competition does not matter: grassland diversity and community composition. The American Naturalist, 171, 777-787.

Litchman, E., de Tezanos Pinto, P., Klausmeier, C.A., Thomas, M.K. \& Yoshiyama, K. (2010) Linking traits to species diversity and community structure in phytoplankton. Hydrobiologia, 653, 15-28.

Liu, X., Swenson, N.G., Zhang, J. \& Ma, K. (2012) The environment and space, not phylogeny, determine trait dispersion in a subtropical forest. Functional Ecology, 27, 264-272.

Losos, J.B. (2008) Phylogenetic niche conservatism, phylogenetic signal and the relationship between phylogenetic relatedness and ecological similarity among species. Ecology Letters, 11, 995-1007.

Losos, J.B., Leal, M., Glor, R.E., de Queiroz, K., Hertz, P.E., Rodriguez Schettino, L., Chamizo Lara, A., Jackman, T.R. \& Larson, A. (2003) Niche liability in the evolution of a Caribbean lizard community. Nature, $\mathbf{4 2 4}$, 542-545

MacArthur, R. \& Levins, R. (1967) The limiting similarity, convergence and divergence of coexisting species. The American Naturalist, 101, 377-385.

Maherali, H. \& Klironomos, J.N. (2012) Phylogenetic and trait-based assembly of arbuscular mycorrhizal fungal communities. PLoS ONE, 7, e36695.

Mayfield, M.M. \& Levine, J.M. (2010) Opposing effects of competitive exclusion on the phylogenetic structure of communities. Ecology Letters, 13, $1085-1093$.

Mouquet, N., Devictor, V., Meynard, C.N., Munoz, F., Bersier, L.-F., Chave, J. et al. (2012) Ecophylogenetics: advances and perspectives. Biological Reviews, 87, 769-785.

Narwani, A., Alexandrou, M.A., Oakley, T.H., Carroll, T. \& Cardinale, B.J. (2013) Experimental evidence that evolutionary relatedness does not affect the ecological mechanisms of coexistence in freshwater green algae. Ecology Letters, 16, 1373-1381.

Posada, D. (2008) jModelTest: phylogenetic model averaging. Molecular Biology and Evolution, 25, 1253-1256.

Prinzing, A., Durka, W., Klotz, S. \& Brandl, R. (2001) The niche of higher plants: evidence for phylogenetic conservatism. Proceedings of the Royal Society B: Biological Sciences., 268, 2383-2389.

Rambaut, A. \& Drummond, A.J. (2009) Tracer v1.5.

Reusch, T.B.H., Ehlers, A., Hammerli, A. \& Worm, B. (2005) Ecosystem recovery after climatic extremes enhanced by genotypic diversity. Proceedings of the National Academy of Sciences, 102, 2826-2831.

Rosch, H., van Rooyen, M.W. \& Theron, G.K. (1997) Competitive effects and response of ten Namaqualand ephemeral plants species at two nutrient levels. South African Journal of Botany, 63, 210-215.

Schoustra, S.E., Dench, J., Dali, R., Aaron, S.D. \& Kassen, R. (2012) Antagonistic interactions peak at intermediate genetic distance in clinical and laboratory strains of Pseudomonas aeruginosa. BMC Microbiology, 12, 40.

Schwaderer, A.S., Yoshiyama, K., de Tezanos Pinto, P., Swenson, N.G., Klausmeier, C.A. \& Litchman, E. (2011) Eco-evolutionary differences in light utilization traits and distributions of freshwater phytoplankton. Limnology and Oceanography, 56, 589-598

Slingsby, J.A. \& Verboom, G.A. (2006) Phylogenetic relatedness limits cooccurrence at fine spatial scales: evidence from the schoenoid sedges (Cyperaceae: Schoeneae) of the Cape floristic region, South Africa. The American Naturalist, 168, 14-27.

Soliveres, S., Torices, R. \& Maestre, F.T. (2012) Evolutionary relationships can be more important than abiotic conditions in predicting the outcome of plant-plant interactions. Oikos, 121, 1638-1648.

Stajich, J.E., Block, D., Boulez, K., Brenner, S.E., Chervitz, S.A., Dagdigian, C. et al. (2002) The bioperl toolkit: perl modules for the life sciences. Genome Research, 12, 1611-1618.

Stamatakis, A., Hoover, P. \& Rougemont, J. (2008) A rapid bootstrap algorithm for the RAxML web servers. Systematic Biology, 57, 758-771.

Swenson, N.G., Enquist, B.J., Thompson, J. \& Zimmerman, J.K. (2007) The influence of spatial and size scale on phylogenetic relatedness in tropical forest communities. Ecology, 88, 1770-1780.

Thuiller, W., Gallien, L., Boulangeat, I., de Bello, F., Münkemüller, T., Roquet, C. \& Lavergne, S. (2010) Resolving Darwin's naturalization conundrum: a quest for evidence. Diversity and Distribution, 16, 461-475.

Tilman, D. (1981) Tests of resource competition theory using four species of Lake Michigan algae. Ecology, 62, 802-815.

Uriarte, M., Swenson, N.G., Chazdon, R.L., Comita, L.S., Kress, W.J., Erickson, D., Forero-Montana, J., Zimmerman, J.K. \& Thompson, J. (2010) Trait similarity, shared ancestry and the structure of neighborhood interactions in a subtropical wet forest: implications for community assembly. Ecology Letters, 13, 1503-1514.

Valiente-Banuet, A. \& Verdú, M. (2007) Facilitation can increase the phylogenetic diversity of plant communities. Ecology Letters, 10, 1029-1036.

Valiente-Banuet, A. \& Verdú, M. (2008) Temporal shifts from facilitation to competition occur between closely related taxa. Journal of Ecology, 96, 489494.

Venail, P. \& Vives, M.J. (2013) Phylogenetic distance and species richness interactively affect the productivity of bacterial communities. Ecology, 94, 2529-2536.

Venail, P., Alexandrou, M.A., Oakley, T.H. \& Cardinale, B.J. (2013) Shared ancestry influences community stability by altering competitive interactions: evidence from an experiment using freshwater green algae. Proceedings of the Royal Society of London Series B: Biological Sciences, 280, 20131548.

Verdu, M., Gomez-Aparicio, L. \& Valiente-Banuet, A. (2012) Phylogenetic relatedness as a tool in restoration ecology: a meta-analysis. Proceedings of the Royal Society of London Series B: Biological Sciences, 279, 1761-1767. 
Violle, C., Nemergut, D.R., Pu, Z. \& Jiang, L. (2011) Phylogenetic limiting similarity and competitive exclusion. Ecology Letters, 14, 782-787.

Received 22 November 2013; accepted 12 May 2014

Handling Editor: James Cahill

\section{Supporting Information}

Additional Supporting Information may be found in the online version of this article:

Figure S1. Unsmoothed molecular phylogeny including 37 common North American freshwater green algae genera and 3 out-groups esti- mated using partial $18 \mathrm{~S}$ ribosomal RNA and rbcl sequences available on GenBank.

Figure S2. Smoothed molecular phylogeny including 37 common North American freshwater green algae genera and 3 out-groups estimated using partial $18 \mathrm{~S}$ ribosomal RNA and rbcl sequences available on GenBank.

Table S1. Contingency Tables of the chi-square tests on the prevalence of facilitation or competition.

Table S2. Raw data including the initial and final densities of invaders. 